MRS Advances (C) 2017 Materials Research Society

DOI: 10.1557/adv.2017.160

\title{
Selective Doping of Quantum Dot Nanomaterials for Managing Intersubband Absorption, Dark Current, and Photoelectron Lifetime
}

\author{
Kimberly Sablon, ${ }^{1}$ Andrei Sergeev, ${ }^{1}$ Xiang Zhang, ${ }^{2}$ Vladimir Mitin, ${ }^{2}$ Michael Yakimov, ${ }^{3}$ \\ Vadim Tokranov, ${ }^{3}$ and Serge Oktyabrsky ${ }^{3}$ \\ ${ }^{1}$ U.S. Army Research Laboratory, Adelphi, Maryland 20783, USA \\ ${ }^{2}$ State University of New York (SUNY) at Buffalo, Buffalo, NY 14260, USA \\ ${ }^{3}$ SUNY Polytechnic Institute, Albany, NY 12203, USA
}

\begin{abstract}
Novel approach to optimize quantum dot (QD) materials for specific optoelectronic applications is based on engineering of nanoscale potential profile, which is created by charged QDs. The nanoscale barriers prevent capture of photocarriers and drastically increase the photoelectron lifetime, which in turn strongly improves the photoconductive gain, responsivity, and sensitivity of photodetectors and decreases the nonradiative recombination losses of photovoltaic devices. QD charging may be created by various types of selective doping. To investigate effects of selective doping, we model, fabricated, and characterized AlGaAs/InAs QD structures with n-doping of QD layers, doping of interdot layers, and bipolar doping, which combines p-doping of QD layers with strong n-doping of the interdot space. We have measured spectral characteristics of photoresponse, photocurrent and dark current. The experimental data show that providing the same electron population of QDs, the bipolar doping creates the most contrasting nanoscale profile with the highest barriers around dots.
\end{abstract}

\section{INTRODUCTION}

Optoelectronic properties of quantum dot nanomaterials were intensively investigated during last two decades. MBE grown structures and colloidal materials demonstrate selective photonelectron coupling associated with electron transitions in QDs. Photon-induced intersubband transitions cover wide spectral range from $\mathrm{THz}$ to infrared and can be effectively controlled by size and form of QDs. Operation of various photoelectron devices, such as THz/Infrared photodetectors and solar cells with intermediate states in QDs, are based on intersubband transitions in QD nanomaterials.

Compared to other optoelectronic nanomaterials, such as quantum well structures and type II superlattices, QD structures are essentially three-dimensional nanomaterials, where processes of photon absorption in QDs are spatially separated from photocarrier transfer along conducting channels formed between dots by nanoscale potential barriers created by charged dots. Therefore QD nanomaterials provide high optoelectronic gain and more flexibility in managing of critical 
kinetic and transport characteristics, such as the photocarrier lifetime and photocarrier mobility. For optimal use of potential of QD detectors the photocarrier lifetime should be adjusted to values required by applications. Long lifetime cannot provide effective sensing in dynamic background. Too short lifetime decreases the sensitivity of the detector.

In QD structures for IR and $\mathrm{THz}$ sensing the photocarrier lifetime is controlled by the charge of QDs [1-3]. The detectors are unipolar devices and probably for this reason up until now unipolar doping only was employed to charge quantum dots. For example, in typical GaAs/InAs QD structures the n-doping of the interdot space was used to provide QD with electrons that may be excited by the IR/THz radiation. However, unipolar doping does not solve all problems. First of all, to increase the photocarrier lifetime the potential barrier around QD should be three-four times larger than $k_{\mathrm{B}} T$. Therefore, at liquid nitrogen temperatures the nanoscale barriers should be at least $0.025 \mathrm{eV}$. The corresponding doping should be above $2-3$ electrons per dot. However, strong unipolar doping changes spectral characteristics of the detector and substantially increases the noise current, which results from the fluctuations in the dark current.

Photocarrier lifetime and noise current are two key optoelectronic parameters of the photodetector. The detector response time is directly given by the photocarrier lifetime and the noise bandwidth is given by the inverse lifetime. A value of detector responsivity is directly proportional to the lifetime. Noise current gives the detector sensitivity. Finally, responsivity and sensitivity determine the maximal operating temperature. Independent control and tuning of the photoresponse and noise characteristics would provide adequate adjustment of the detector to application requirements and substantially increase the commercial potential of QD photodetectors.

\section{THEORY}

The proposed QD nanomaterials with bipolar doping are expected to provide long and controllable photocarrier lifetime and, therefore, enhanced photoresponse, together with independently manageable dark and noise currents, which determine the detector sensitivity and the open circuit voltage of QD solar cells. QD structure with bipolar doping combines p-doping of the quantum dot layers with the acceptor concentration per dot, $n_{a}$, and n-doping of the interdot space with the dopant concentration per dot, $n_{d}$. The electrons from donors populate QDs and build charged complexes with acceptors. As a result, the charge of QDs is equal to the number of donors per dot, $n_{d}$, but the number of carriers in dot is $\left(n_{d}-n_{a}\right)$. In the limit $n=p>>1$, carriers in dots are practically absent, while QD are strongly charged and surrounded by huge potential barriers (see Fig. 1).

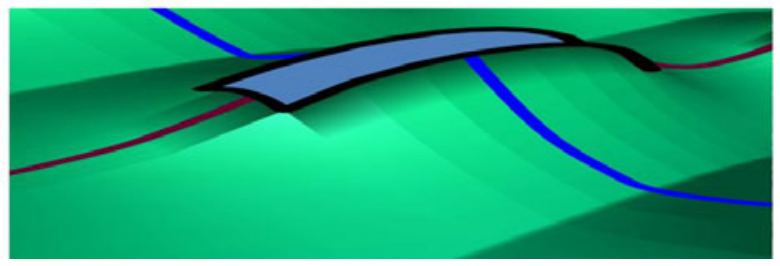

Figure 1. Nanoscale potential barriers around charged quantum dot. 
The thermally generated carrier density may be expressed in terms of the equilibrium carrier concentration in dots as follows:

$$
n_{\mathrm{th}}=\left(n_{d}-n_{a}\right) N_{\mathrm{d}} \exp \left[\left(E_{\mathrm{C}}-E_{\mathrm{L}}\right) / k T\right],
$$

where $E_{\mathrm{C}}$ and $E_{\mathrm{L}}$ are the energy of conducting and localized electron states correspondingly and $N_{\text {D }}$ is the dot concentration.

The intrinsic generation-recombination noise is determined by the kinetics of thermal carriers. To obtain the noise current in the photoconductor, let us assume that without light there are $N_{\text {th }}$ thermally excited charge carriers in the channel. Then, the dark current is given by

$$
J_{d c}=e N_{t h} / \tau_{t r}=e n_{t h} S / \tau_{t r} \propto\left(n_{d}-n_{a}\right),
$$

where $S$ is the device square and $\tau_{t r}$ is the electron transit time.

The photocarrier lifetime exponentially increases due to potential barriers around negatively charged quantum dots [1-5],

$$
\frac{1}{\tau_{\ell}}=\pi N_{\mathrm{D}} a^{3} \frac{1}{\tau_{\varepsilon}} \exp [-e \phi(Q) / k T] \propto \exp \left(-\frac{\phi\left(n_{d}\right)}{k_{B} T}\right)
$$

where $a$ is the QD radius, $1 / \tau_{\varepsilon}$ is the inelastic electron-phonon relaxation rate, and $\phi$ is the potential at the dot boundary. The exponential factor describes the effect of potential barriers on capture processes, where the potential barrier $\phi$ is proportional to the charge of the dot $Q=e n_{d}$.

Thermal fluctuations in number of thermally generated electrons, $N_{\text {th }}=n_{t h} S$, lead to the generation-recombination (GR) noise current. Taking into account that the average fluctuation of $N_{\mathrm{th}}$ is $N_{\mathrm{th}}{ }^{1 / 2}$ and the noise bandwidth of GR processes, $B=1 /\left(2 \tau_{\ell}\right)$, the GR noise current is given by

$$
J_{G R}=\frac{e \sqrt{2 N_{t h}}}{\tau_{t r}} \frac{1}{\sqrt{B}}=2 \sqrt{e J_{d c} \gamma} \propto\left(\left(n_{d}-n_{a}\right) \cdot \exp \left(-\frac{\phi\left(n_{d}\right)}{k_{B} T}\right)\right)^{1 / 2}
$$

where $\gamma=\tau_{\ell} / \tau_{t r}$ is the photoconductive gain.

The responsivity of the photodetector is proportional to the photoconductive gain,

$$
R \equiv \frac{J_{p h}}{P}=\frac{e \eta \tau_{\ell}}{h v \tau_{t r}}=\frac{e \eta \gamma}{h v} .
$$

Equations (1) - (5) show that the proposed bipolar doping provides strong decrease of the thermally generated carrier density, $n_{\text {th }}$ (Eq. (1)), and exponential increase of the photoelectron lifetime (Eq. (3)). Two parameters of bipolar doping (donor and acceptor concentrations) provide the decoupled control of the photoelectron lifetime and noise characteristics of the detector.

Our recent investigations of QD solar cell (GaAs solar cells with various InAs QD media) have demonstrated that the main cause of reduction of the open circuit voltage, $V_{\mathrm{OC}}$, is the fast capture of photoelectrons from the GaAs conduction band (CB) to localized states in QDs [6]. We derived condition for the $V_{\mathrm{OC}}$ recovery

$$
\tau_{\ell} \geq \tau_{R},
$$


where $\tau_{\mathrm{R}}$ is the radiative recombination time in the interdot layers. As the photoelectron capture into QDs is mainly realized via the wetting layers (WLs), we substantially reduced the WLs using two monolayer AlAs capping of QDs. In the structures with reduced WLs, the direct CBto-QD capture was further suppressed due to charging of QDs [6]. The bipolar doping allows one to increase the photoelectron lifetime independently from the dark current and, in accordance with Eq. 6, to reach voltage recovery without WL reduction and the dark current increase.

\section{EXPERIMENT}

To investigate effects of selective doping, we model, fabricated, and characterized AlGaAs/InAs QD structures with n-doping of QD layers, doping of interdot layers, and bipolar doping, which combines p-doping of QD layers with strong n-doping of the interdot space. A set of three samples with variable doping level was grown using EPI GEN II MBE system on $\mathrm{n}^{+}-$ GaAs substrates. The samples included the following layers: $450 \mathrm{~nm}$ thick n-GaAs:Si $\left(2 \times 101^{8}\right.$ $\mathrm{cm}^{-3}$ ), $50 \mathrm{~nm}$ of undoped $\mathrm{GaAs}, 150 \mathrm{~nm}$ of undoped $\mathrm{Al}_{0.22} \mathrm{Ga}_{0.78} \mathrm{As}$, active QD structure with total $0.5 \mu \mathrm{m}$ thickness, $75 \mathrm{~nm}$ of undoped $\mathrm{Al}_{0.22} \mathrm{Ga}_{0.78} \mathrm{As}$, $50 \mathrm{~nm}$ of undoped $\mathrm{GaAs}$, and top $300 \mathrm{~nm}$ thick contact n-GaAs: $\operatorname{Si}\left(2 \times 10^{18} \mathrm{~cm}^{-3}\right)$. Each sample contained 10 layers of self-assembled InAs QDs embedded into $\mathrm{Al}_{0.2} \mathrm{Ga}_{0.8} \mathrm{As}$ matrix as an active structure. Substrate temperature was maintained at $475{ }^{\circ} \mathrm{C}$ for optimum QD formation throughout the growth of the active structure. Total of 1.9 monolayers of InAs was deposited per QD layer, with critical thickness of 2D-3D transition being about 1.5 monolayers. Ripening time of 5 seconds was used before capping a QD sheet with $\mathrm{AlGaAs}$ layer. N-type dopant $(\mathrm{Si})$ was introduced into $\mathrm{Al}_{0.2} \mathrm{Ga}_{0.8} \mathrm{As}$ matrix between QD layers while p-type dopant (carbon from $\mathrm{CBr}_{4}$ source) was introduced directly during InAs growth for in-QD incorporation to ensure acceptors being contained directly within InAs QDs. Schematic diagram of QDIP structure showing location of dopants within QD structure is shown in Fig. 2, and amount of dopant in different samples normalized to QD density is summarized in Table I.

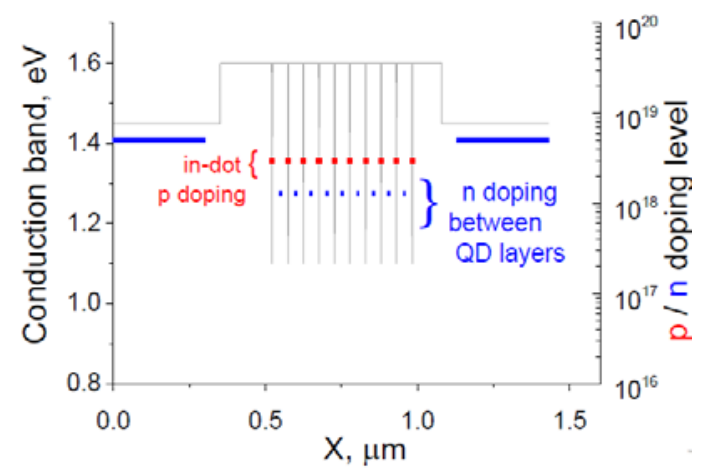

Figure 2. Schematic of QDIP structure showing location of n- and p-doping relative to QDs. 
Table I. Selective doping of QD structures

\begin{tabular}{lcc}
\hline $\begin{array}{l}\text { Sample } \\
\text { ID }\end{array}$ & $\begin{array}{c}\text { n-doping, } \\
\text { Donors per QD } \\
\text { (sheet density, cm } \text { cm }^{-2}\end{array}$ & $\begin{array}{c}\text { p-doping, } \\
\text { Acceptors per QD }\end{array}$ \\
\hline $4 \mathrm{e} 0 \mathrm{p}$ & $4\left(2 \times 10^{11}\right)$ & 0 \\
$4 \mathrm{e} 2 \mathrm{p}$ & $4\left(2 \times 10^{11}\right)$ & 2 \\
$7 \mathrm{e} 3 \mathrm{p}$ & $7\left(3.5 \times 10^{11}\right)$ & 3 \\
\hline
\end{tabular}

Density of surface QDs measured on a test sample, as shown in Fig. 3 was used for calibration of QD concentration and evaluations of the carrier density and QD charging.

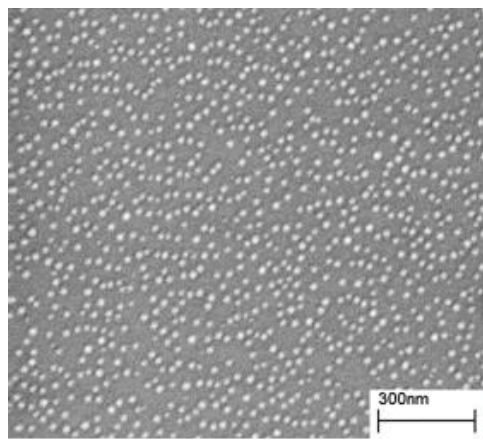

Figure 3. Top-view SEM of QD structure, with estimated QD density is $5 \times 10^{10} \mathrm{~cm}^{-2}$.

After growth and preliminary characterization, the structures were processed into QDIP devices by deposition of AuGeNi Ohmic contacts: a blanket layer on the back of the substrate as a common contact and a lift-off patterned contact on the front surface. Wet chemical etch using $\mathrm{H}_{2} \mathrm{SO}_{4}+\mathrm{H}_{2} \mathrm{O}_{2}$ was utilized to separate individual devices on the common substrate.

Measurements of photoluminescence response at low power laser excitation (below 1 $\mathrm{W} / \mathrm{cm}^{2}$ ) were performed for characterization of electron energy levels and their filling due to selective doping. The results presented in Fig. 4 allow us to identify three interband electron transitions. The characteristic energies of these transitions $(1.16, \sim 1.24$, and $1.32 \mathrm{eV})$ are practically independent on selective doping. As in a number of previous works, the level structure show practically equidistant excited level positions. The total level spacing, $\Delta E=\Delta E_{\mathrm{e}}+$ $\Delta E_{\mathrm{h}}$, directly determined from photoluminescence experiments is $\sim 80 \mathrm{meV}$. The specific equidistant positions of energy levels may be associated with the quasi-parabolic form of the confinement potential in QDs. In this model, the spacing ratio, $\Delta E_{\mathrm{e}} / \Delta E_{\mathrm{h}}$, is given by $\left(m_{\mathrm{h}} / m_{\mathrm{e}}\right)^{1 / 2} \approx 4$, which is in reasonable agreement with the scope of various experimental results. Using this model we obtained: $\Delta E_{\mathrm{e}} \approx 64 \mathrm{meV}$ and $\Delta E_{\mathrm{h}} \approx 16 \mathrm{meV}$. Therefore, the electron transitions in QDs significantly exceed the thermal energy and cannot be induced by thermal phonons. 

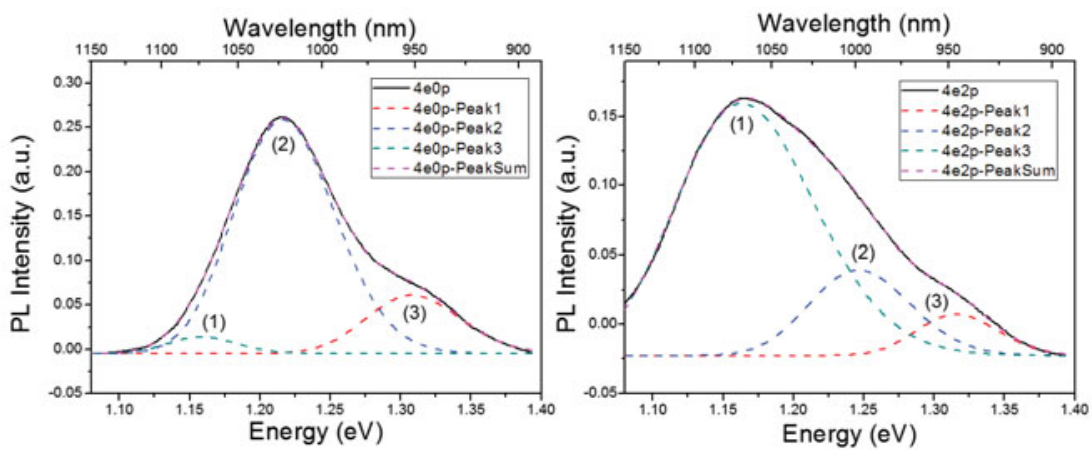

Figure 4. Photoluminescence from $\mathrm{QD}$ in $4 \mathrm{e} 0 \mathrm{p}$ and $4 \mathrm{e} 2 \mathrm{p}$ structures.

The results of the dark current measurements are presented in Fig. 5. As it is expected, the QD structures with $4 \mathrm{e} 0 \mathrm{p}$ and $7 \mathrm{e} 3 \mathrm{p}$ show close values of the dark currents because of the same electron filling of QDs, 4 electrons per dot, in these structures. In the structure with $4 \mathrm{e} 2 \mathrm{p}$ doping, which provides $\sim 2$ electrons per dot, the equilibrium Fermi level moves further from the conduction band and the dark current is significantly smaller.

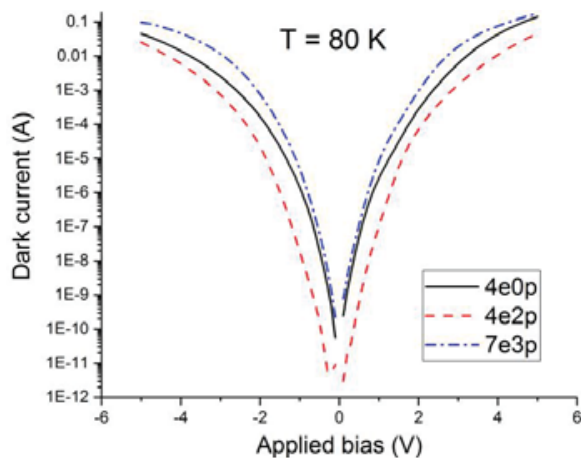

Figure 5. Dark current in 4e0p, 4e2p, and 7e3p QD structures.

Finally, using FTIR we have measured the spectral characteristics of photoresponse, which are presented in Fig. 6. The photoresponse data are consistent with other results. As well as the photoluminescence data in Fig. 4, the spectral characteristics of the photoresponse of 4e0p, $4 \mathrm{e} 2 \mathrm{p}$, and 7e3p QD structures are also independent on doping. The peak of the photoresponse is observed at $\sim 270 \mathrm{meV}$, which is $\sim 4 \Delta E_{\mathrm{e}}$, as it is usually observed in QD photodetectors. The photoresponse is enhanced by $\sim 25 \%$ in the $7 \mathrm{e} 4 \mathrm{p}$ structure with strong bipolar doping. 


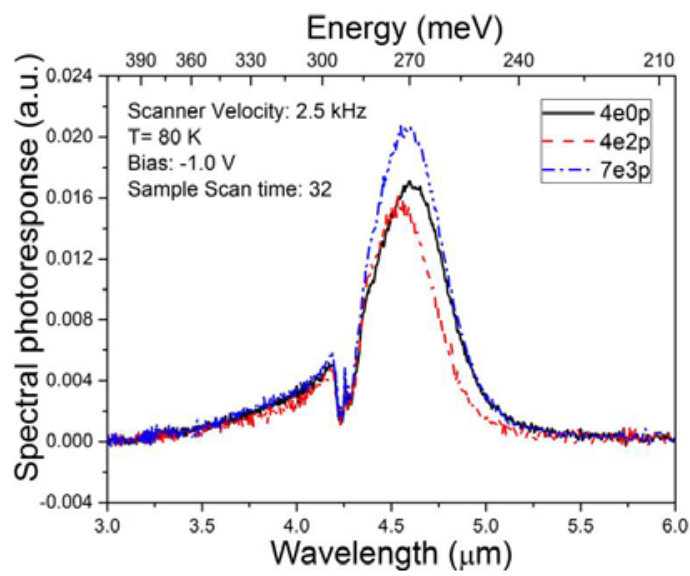

Figure 6. Spectral characteristics of photoresponse in $4 \mathrm{e} 0 \mathrm{p}, 4 \mathrm{e} 2 \mathrm{p}$, and 7e3p QD structures.

\section{CONCLUSIONS}

Charging of quantum dots is efficient tool to control photoelectron kinetics via nanoscale potential profile [1,6-8]. Traditional doping of interdot layers provides QD charging simultaneously with QD filling. In this case, the kinetic characteristics (photoelectron lifetime) and equilibrium thermodynamic characteristics (dark current, absorption coefficient) cannot be independently optimized for specific applications. As it is demonstrated in this work, the bipolar doping, which includes the compensating p-doping of QD layers, allows for independent management of equilibrium and kinetic properties of optoelectronic QD nanomaterials. The experimental results presented in Fig. 6 show that increase of electron charging by 3 electrons per dot without changes in electron filling of QDs increases the photoresponse by $\sim 25 \%$, while the dark current does not change essentially at low voltages (Fig. 5). However, this increase of photoresponse is by one order in magnitude below than it may be expected from Eqs. 3 and 5 . Such significant difference has clear explanation. In this work we doped the QD layers, not QDs. Doping of QD layers creates strong nanoscale barriers only in the direction perpendicular to QD layers. In QD layers the potential barriers between QDs are negligible. Development of technologies for direct doping of QDs will strongly increase photoresponse and enhance functionalities of QD nanomaterials.

\section{ACKNOWLEDGMENTS}

This research was supported by ARL. AS acknowledges support by NRC. 


\section{REFERENCES}

1. K. A. Sablon, J.W. Little, V. Mitin et al., Nano Letters 11, 2311 (2011).

2. V. Mitin, A. Antipov, A. Sergeev et al., Nanoscale Research Letters 6, 21 (2011).

3. K. A. Sablon, J. W. Little, A. Sergeev et al., J. Vac. Sci. Technol. 30, $04 \mathrm{D} 104$ (2012).

4. V. Mitin, A. Sergeev, N. Vagidov et al., Infrared Physics \& Technology 59, 84-88 (2013).

5. K. Sablon, J. Little, N. Vagidov et al., Applied Physics Letters 104, 253904 (2014).

6. A. Varghese, M. Yakimov, V. Tokranov et al., Nanoscale 8, $7248-7256$ (2016).

7. A. Sergeev, N. Vagidov, V. Mitin, and K. Sablon, "Charged quantum dots for high efficiency photovoltaics and IR sensing," Future Trends in Microelectronics, ed. S. Luryi, J. Xu, and A. Zaslavsky (Wiley-IEEE Press, 2013) pp. 244-253.

8. K. A. Sablon and A. Sergeev, "Emerging PV Nanomaterials: Capabilities versus Recombination Losses," High-Efficiency Solar Cells: Physics, Materials, and Devices, ed Xiaodong Wang and Zhiming M. Wang (Springer Series in Materials Science, 2014) pp. 85114. 